九州大学学術情報リポジトリ

Kyushu University Institutional Repository

\title{
A Novel Traveling Salesman Problem Solution by Accelerated Evolutionary Computation with Approximated Cost Matrix in Industrial Application
}

Pei, Yan

Graduate School of Design, Kyushu University

Takagi, Hideyuki

Faculty of Design, Kyushu University

http://hdl. handle. net/2324/1906106

出版情報: Proceedings of the 2011 International Conference of Soft Computing and Pattern Recognition (SoCPaR), pp. 39-44, 2011-10-16. IEEE

バージョン:

権利関係：(C)2011 IEEE 


\section{A Novel Traveling Salesman Problem Solution by Accelerated Evolutionary Computation with Approximated Cost Matrix in an Industrial Application}

\author{
Yan Pei \\ Graduate School of Design, Kyushu University \\ Fukuoka, Japan \\ peiyan@ieee.org
}

\author{
Hideyuki Takagi \\ Faculty of Design, Kyushu University \\ Fukuoka, Japan \\ http://www.design.kyushu-u.ac.jp/ takagi
}

\begin{abstract}
We propose an industrial technological solution for the traveling salesman problem (TSP) by using the approximated cost matrix and an accelerated evolutionary computation (EC) algorithm. The cost matrix used by theoretical research on TSP mostly is the Euclidean distance between cities, which is not proper to the real condition in the industrial product's application. In this paper, we propose an approximation approach on cost matrix based on the geographic information data, so that it approaches to the actual cost matrix. Slow convergence is the main issue of EC. We propose an accelerating EC convergence approach by Lagrange interpolation method to approximate the EC search space landscape, and do a local search near the related best individuals' region. The experimental result shows that the EC convergence is accelerated, and this acceleration approach is also used in an actual TSP application in a vehicle navigation system, in which the product performance is improved by the accelerated EC approach with the approximated cost matrix.
\end{abstract}

Keywords-traveling salesman problem; geographic information data; evolutionary computation; search space landscape information; convergence acceleration

\section{INTRODUCTION}

The traveling salesman problem (TSP) is a classical combinatorial optimization problem, which is also a challenging mathematical problem, i.e., a NP-complete problem (NP, i.e. nondeterministic polynomial time). That is, the TSP asks for finding a tour through a finite set of $n$ cities and a cost matrix between each pair. Each city is visited exactly once. When returning to the starting city, the total travel cost must be minimized. This simple description problem has not been solved completely yet since the day it was proposed, because its computational complexity rises exponentially by increasing the number of cities. An alternative solution of TSP has been to apply a proper suboptimal algorithm in many of current TSP applications such as bioinformatical data analyses [1], memory accesses reordering [2], navigation [3], task schedules [4] and data partitioning [5].

There are two main research directions to solve the TSP. One is by the conventional combinatorial optimization approach, such as the Lin-Kernighan algorithm [6], tabu search [7], local search [8], and $A^{*}$ graph search [9]. The other main approach is artificial intelligence technology, such as artificial neuron network (NN) [10]-[18] [31], evolutionary computation (EC) [19]-[27], Simulated Annealing (SA) [28] and ant colony algorithm [29] [30].
The Lin-Kernighan algorithm [6] was developed about forty years ago, but it remains one of the best heuristics search algorithms for the TSP until now. So for this research direction, a more powerful optimal combination technology is needed and further theoretical mathematics development is necessary to improve progress of TSP solution. In forty years, however, few innovational algorithms have been proposed. The main focused point of this research direction is the fusion technology to combine existent methods to obtain a better TSP performance.

There are two main topics in fusion technology. One is artificial intelligence algorithm fusion with the conventional optimal combination technology. Reference [7] implements the crossover operator of the genetic algorithm with tabu search, and indicates that different strategies are the key points for the efficient tabu search. Another considered aspect that has been focused on is the technique to reduce the complexity of a large-scale TSP instance for decomposing or partitioning it into smaller sub problems. The other is fusion of artificial intelligences, such as NN + EC, NN $+\mathrm{SA}$, etc. For using the NN to solve the TSP, there are mainly types such as Hopfield neural network [10] [13], adaptive neural network [11] [18], self-organizing map [12] [16] [17] [31], chaotic neural network [14]. Reference [15] uses a feedback structure similar to Hopfield-type neural networks and a competitive training algorithm similar to the Kohonen-type self-organizing maps, fusing those two neural networks together to use each efficient feature to improve the TSP. It is a powerful tool to use the EC for TSP in recent decades and fusion technology is also an important method to deal with the TSP in EC. Simulated annealing and ant colony algorithm are principal tools used to make up for the drawback of the other optimization technology. Reference [32] is a review literature by D.S. Johnson, it summaries up the main solution and technology to solve TSP.

The drawback of the conventional TSP solution by EC is the slow convergence, so many novel EC methods were proposed. There are three main research topics. The first is to design TSP specific operators [33]-[35]. The second is to incorporate local search or fuse with other optimization technology [36] [37], and the third is to maintain population diversity [36] [38]. The purposes of those methods are to keep the diversity of the individuals to accelerate EC convergence, which concerns distribution of the individuals in the search space to avoid premature, as well as the 
balance to exploit global optimum in local and to explore new searching areas in the whole search space.

Much theoretical research on TSP has been described in thousands of papers in the academic community. However, the application-oriented research work on TSP industrial application has been less reported in recent decades. This paper proposes an industrial solution on TSP in a vehicle navigation system, which uses an accelerated EC convergence approach by Lagrange interpolation and defines an approximated cost matrix solution based on the geographic information data. This paper is an application-oriented work in intelligence transportation, in which there are two original contributions. On the one hand, it reviews about forty manuscripts on the conventional TSP acceleration solution and summarizes up the geographic information data in industrial development in the last two decades. On the other hand, it uses an interpolation approach to approximate EC search space landscape to accelerate EC convergence in a practical industrial solution of TSP and gives a practical solution on defining an approximated cost matrix based on geographic information data, which approximates the real cost between the two waypoints in a vehicle navigation system.

The remainder of this paper is structured as follows. Section II gives a normal description of TSP and introduces the conventional TSP solution in a vehicle navigation system. Section III reviews the geographic information data development and data format in the geographic information system (GIS). It especially concentrates on the OriginDestination Routing Data (ODRD) related to our cost matrix approximation approach, and a concrete cost approximation solution is described based on the ODRD. Section IV shows the EC acceleration approach of TSP in a vehicle navigation system and investigates its important issues that need to be solved in practice. The evaluations are made in Section V, which gives six sample results with simulation data. Section VI gives an overview on some further research issues, and several open topics are discussed.

\section{Conventional Traveling Salesman Problem Solution in a Vehicle NaVigation System}

\section{A. Traveling Salesman Problem Model}

TSP is described as a set of cities (Equation (1)),

$$
C=c_{0}, c_{1}, \ldots, c_{n}
$$

and a cost matrix between each pair of cities (Equation (2)),

$$
\mathbf{M}=\left(\begin{array}{ccc}
A_{\left[c_{0}\right]\left[c_{0}\right]} & \ldots & A_{\left[c_{0}\right]\left[c_{n}\right]} \\
\vdots & \ddots & \vdots \\
A_{\left[c_{n}\right]\left[c_{0}\right]} & \ldots & A_{\left[c_{n}\right]\left[c_{n}\right]}
\end{array}\right)
$$

the TSP asks to find a path through all cities by visiting each city only once. It requires to set up the visiting order as $Q$, and to return to the city of origin such that the total travel cost is minimized. This is given by Equation (3).

$$
\min \left(\sum_{i=0}^{n} A_{[Q(i)][Q(i+1)]}+A_{[n][0]}\right)
$$

The solution of TSP is to find out the order sequence $Q$. Because of the cost matrix is symmetric in theory, the number of solutions of TSP with $N$ cities is $O[N ! / 2]$. But in a real-world application, such as a vehicle navigation system, the cost matrix is non-symmetric, so that the solution of TSP is $O[N !]$. A special requirement of TSP in a vehicle navigation system is that the vehicle position and the destination points are not changed. Due to this kind of requirement, there are $N$ points in the vehicle navigation system, and the number of TSP solution count is $O[(N-2) !]$.

\section{B. Conventional TSP Solution in Vehicle Navigation System}

Some TSP solutions are investigated in section I. The main theoretical research approaches are focused on the LinKernighan algorithm and its improvement, neural networks and evolutionary computation. There are few reports on TSP solutions in industrial products, such as location based service system, vehicle navigation system, etc. Because of the limited resources in industrial production and the real-time requirement, e.g., as in embedded systems, the approximation algorithm of TSP in a vehicle navigation system, the so-called nearest waypoint algorithm (NWA) is proposed.

The nearest waypoint algorithm defines the search principle that finds the next waypoint near the current position by measuring the Euclidean distance between two points. For example, in a vehicle navigation system, the vehicle position is the fixed one, so we need to begin the search in this point. The next best candidate point is the least Euclidean distance point, but this candidate point is excluding the destination point. By this principle, we can obtain the entity waypoint sequence until the destination is found.

The running time growth rate of TSP is $O((N-2)$ !) in a vehicle navigation system, but the abovementioned NWA is just $O(N-2)$. Although some solutions by NWA are worse, it is a suboptimal algorithm and alternative solution in the industrial applications. The performance is improved from $O(N$ !) to $O(N)$. The NWA loses the solution quality to obtain a better performance, so it needs the improved algorithm to give a relative better solution in the vehicle navigation system.

\section{COSt MATRIX APPROXIMATION APPROACH}

\section{A. State-in-art of Geographic Information Data}

The geographic information data (GID) is the fundament of a geographic information system research and application. Two issues are considered in the GID. One is the data information integrality, which is constructed to express the real-world information in its application domain. It can represent real objects integrally, such as roads, land use, elevation, trees, waterways, etc, and its format is common in all the data expression approaches. The other is the data information usability, which can be easy to utilize in all the related geographic information system applications, and the information is not redundant.

The design proposal of data store is by level and by mesh, which is the primary GID management and store approach. For data store by level, due to the information granularity, GID can be expressed into a different level, and 
the information quantity in the each level is compressed with a certain principle and its granularity. For data store by mesh, for convenient management of GID, stores the data by mesh in each level, and the mesh can be separated into smaller ones according with a certain data quantity.

Most industrial GID is developed by professional companies, such as NAVTEQ, Zenrin, MapInfo, etc. The data format standard is investigated and designed by those big institutions separately. The geographic data format (GDF), an European standard created by Committee European de Normalization (CEN), is emerging as the de facto international standard for exchanging navigable databases. GDF has multiple versions, and the latest is version 5.0, which prevents usage of a single GDF compiler worldwide to serve all map suppliers. The GDF conceptual data model comprises three entities: levels, attributes, and relationships. Some application investigations based on GID were reported in Reference [39] [40].

\section{B. Origin-Destination Routing Data Format}

For accelerating the routing calculation for a fast convergence, a so-called original-destination routing data (ODRD) format has been proposed and developed in geographic information data format, which stores every mesh relationship between the original and destination mesh. For a certain original and destination pair, we can obtain their mesh ID pair, and the original-destination routing data sequence is indexed by this ID pair, and then, when running a routing calculation for a fixed original and destination, the search engine uses the data mentioned in sequence of originaldestination routing data rather than all the data. It decides the data selection range to reduce the data accessing time to accelerate the routing calculation.

\section{Cost Matrix Approximation Approach by Geographic Information Data}

In the TSP theoretical research, we consider the Euclidean distance as simulation in cost matrix among the cities. However, in the real-world geographic information system applications, the Euclidean distance is not exact and cannot be used instead of the real cost between two cities. Therefore a promising research topic is how to obtain the approximated cost as close to the real cost by the geographic information data. A good solution is to use ODRD, which stores the usable data between the original and destination data pair. We can use this kind of data to approximate the cost between two points in the real geographic information system application [41].

From the ODRD, we can obtain a set of mesh, which stores the meshes that can be passed by the path from original to destination. The shadow meshes are the meshes that can be passed from $A$-point to $B$-point (Figure. 1 (a)). Next we select the geometrical center points of each mesh to obtain the approximated cost (Figure. 1 (b)). For an easy calculation of the cost between points in the Cartesian coordinate, we transfer a certain angle in the coordinate so that we can let the new line through $A$ and $B$ be as the new $X$ axes (Figure. 1 (c)). We cut the $X$ axes into small pieces with a fixed distance, which is probably the

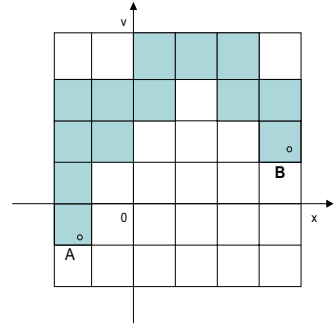

(a)

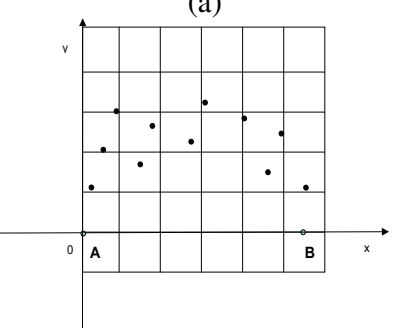

(c)

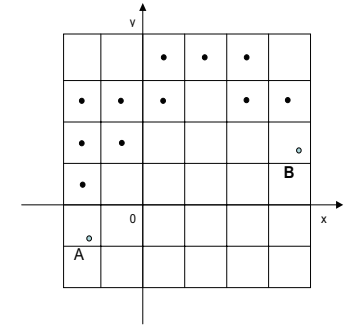

(b)

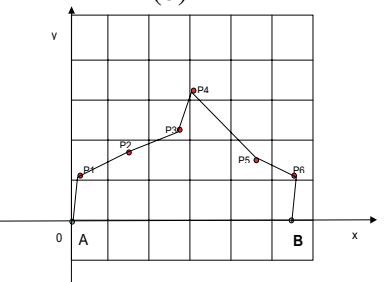

(d)
Figure 1. Cost matrix approximation approach, the meshes stored in ODRD are shown in (a), (b) shows the center points of each mesh, (c) is the coordinate transformation result, connecting center points as the approximation cost in (d).

length of the mesh side, and select the nearest points as the candidate points. We then connect those points, and the curve's length is the approximation cost between the $A$ point and $B$ point (Figure. $1(\mathrm{~d})$ ). We use this kind of approach to approximate any cost between two points to establish the cost matrix in the geographic information system application by the ODRD.

\section{TSP SOLUTION BY ACCELERATED EVOLUTIONARY COMPUTATION APPROACH}

\section{A. TSP Solution by EC in Vehicle Navigation System}

Evolutionary computation algorithms (ECs) are power tools to solve the combinatorial optimization problem such as TSP, but the drawback of the EC application is its slow convergence. It is a promising research direction to use the fitness landscape information to accelerate ECs. The approximation approach can obtain the fitness landscape information, where it becomes easier to find elite from the regression space approaching to the global optimum. It is not the actual global optimum, but may be a neighbor around the global optimum in the original search space. In the next interaction, it is higher possible to obtain the actual global optimum from this neighbor point.

Many functional requirements of a vehicle navigation system can be abstracted from the TSP model. However, existent TSP models need to be modified to adapt the actual application. In the vehicle navigation system, the customer can set many waypoints, but the vehicle position and the destination are the fixed points in one route planning. So when we design the ECs to solve the TSP in vehicle navigation system, we need to consider this characteristic in the individual coding, EC operations, fitness function design, etc. 


\section{B. Fitness Landscape Information Approximation Approach}

The Lagrange interpolation polynomial shows the nonlinear and uniqueness character in the interpolation polynomial. It is a good tool to obtain regression space of the fitness landscape information. Equation (4) is the $n$ degree Lagrange interpolation benchmark function.

$$
l(x)=\prod_{i=1, i \neq k}^{n} \frac{\left(x-x_{i}\right)}{\left(x_{k}-x_{i}\right)}
$$

The $\mathrm{n}$ degree Lagrange interpolation polynomial shows in Equation (5).

$$
L_{n}(x)=\sum_{k=0}^{n} l_{k}(x) y_{k}, \quad i=0,1, \ldots, n
$$

In this paper, we use the two degree Lagrange interpolation polynomial to simplify search space to obtain the fitness landscape information, and use the three relative best fitness individuals as the interpolation point. The concrete interpolation polynomial is the Equation (6), and the interpolation handling is to find out the parameters of Equation (6).

$$
L(x)=\sum_{k=1}^{3}\left\{\prod_{i=1, i \neq k}^{3} \frac{\left(x-x_{i}\right)}{\left(x_{k}-x_{i}\right)}\right\} y_{k}
$$

\section{Accelerating Evolutionary Computation Approach}

The acceleration approach chooses new elite from a simplified search surface by Lagrange two degree polynomial interpolation. That means it does a local search in the original search space of the sampling points in the relative best fitness area. It is hoped that the global optimum is around the area and we can obtain it by chance [42]. The regression search space by Lagrange two degree polynomial interpolation is nonlinear space. Considering the space feature, it is easy to obtain the stationary point by the gradient information, so we obtain the new elite from the stationary point in the regression search space.

There is one more individual in a population after inserting new elite into original search space. For keeping a certain size of a population, we must remove one individual from the original population. The acceleration approach selects the worst fitness individual from the original search space, and removes it. On the one hand, the worst one is hard to be selected by the roulette wheel sampling selection approach, and on the other hand, there is a small possibility that the global optimum is near the worst fitness individual. For the viewpoint of keeping a fixed number of population size, the influence of the removing method is less on the original search space.

When we obtain the new elite in the stationary point of the regression search space, it may be not a correct sequence solution of TSP or it may escape from the original search space. We thus need to find an alternative solution as the new elite onto the next search space. In the case of an incorrect sequence, we should find the nearest solution in the regression space as the alternative one. For the escape case, we need to make sure it is in the search space and find the nearest solution.

\section{Experimental Simulation Evaluation}

\section{A. Experimental Setting}

To evaluate the proposed approach, we set up 6 TSP simulation models with the approximated cost matrix with the size of $7 * 7$. This simulates a model with five waypoints, one original point and one destination point in a vehicle navigation system. The approximated cost matrix comes from actual ODRD in a vehicle navigation system. For each simulation model, we test each case by 20 generations for 30 trials running and obtain each generation's best fitness average value to compare the performances of the normal GA (GA-N) and accelerated GA (GA-LR) (see Figure. 2). The GA detail parameters setting is in Table I. After this we obtain the average effectiveness time of each approach by applying the t-test to test the hypothesis analysis.

Table I

GA PARAMETERS SETTING

\begin{tabular}{ll}
\hline Item & Value or Setting \\
\hline Coding & Integer Number \\
\# of Generation & 20 \\
Population Size & 20 \\
Selection & Roulette Wheel Selection \& Elite Strategy \\
Crossover & One-point \\
Crossover Rate & $90 \%$ \\
Mutation Rate & $10 \%$ \\
\hline
\end{tabular}

\section{B. Hypothesis Analysis}

Before applying the t-test, we confirmed whether the average effectiveness of GA-N and GA-LR followed a normal distribution by using the Jarque-Bera test, and the results proof that they follow the normal distribution. Before applying t-test, we assume that variances of GA-N and GALR were equal. We applied a T-test to GA-N with GA-LR to check whether they were significantly different or not.

Table II

HYPOTHESIS ANALYSIS RESULTS

\begin{tabular}{lcc}
\hline Statistic Value & GA-N & GA-LR \\
\hline Mean & 14.5 & 54.17 \\
Variance & 149.9 & 529.77 \\
Mean's Difference & 39.67 \\
Average Variance & 339.83 \\
Root of Average Variance & 8.43 \\
T-Test Distribution Value $(\alpha / 2<0.005)$ & 15.42 \\
\hline
\end{tabular}

Form the T-test result, we could obtain the mean, the variance, and the t-test distribution value in the significance level of 0.005 accordance with the following Equation (7). The statistical variables are in Table II.

$$
T=t_{\alpha / 2}\left(n_{1}+n_{2}-2\right) \sqrt{\frac{1}{n_{1}}+\frac{1}{n_{2}}} s^{*}
$$

\section{Performance Analysis}

We analyzed the performance of the proposed approach from a T-test result (Table II.) and average convergence 


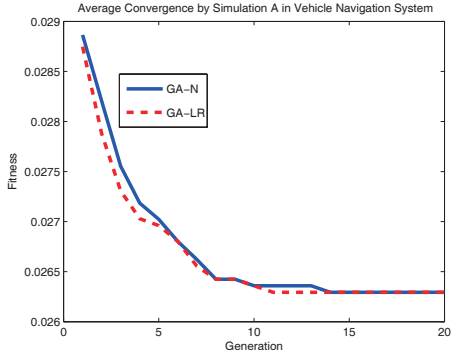

(a)

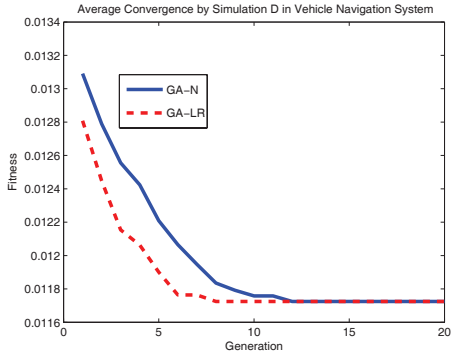

(d)

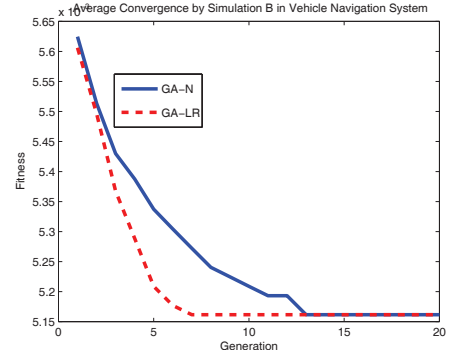

(b)

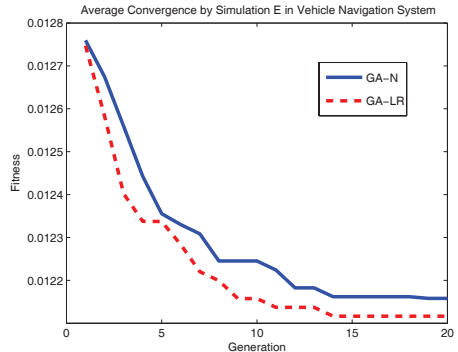

(e)

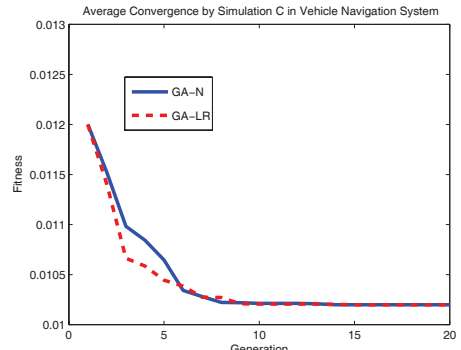

(c)

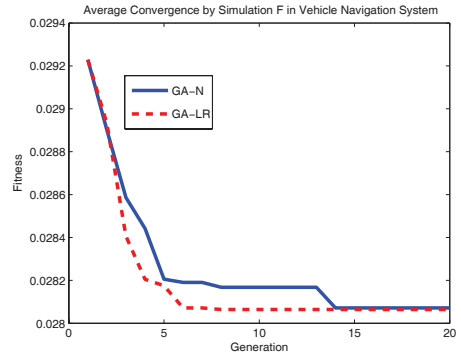

(f)

Figure 2. Six simulation average convergence curves for 30 trails runs, where GA-N and GA-LR mean normal GA and accelerated GA with search space landscape approximated by Lagrange interpolation approach. The proposed approach converges better than normal GA $(p<0.005)$.

comparison (Figure. 2). The effectiveness and reliability of our approach is a key aspect that we considered.

The T-test result showed that the distribution value was less than the mean's difference at a significance level of 0.005 , i.e., $39.67>15.42$. From this we can conclude that the average effectiveness times of the GA-LR and GA-N were not equal $(p<0.05)$. And from average convergence of best fitness comparison (Figure. 2), it shows that GA-LR was more efficient than GA-N in all six simulation cases, and it indicates that the proposed EC acceleration approach can be efficient in the a TSP industrial application.

However, the acceleration performances were different because of the cost matrix setting, which made a different discrete search space. Through analyzing the statistical data, we found that a new elite selection is a key step to the acceleration approach performance. If the elite escape from the search space, the candidate by the nearest point usually is not a better solution. Our proposed approach is a practical and high-performance solution in the TSP industrial application with promising business potential, especially in the industrial products such as the vehicle navigation systems and location based service systems.

\section{CONCLUSION AND FUtURE WORK}

Theoretical research on TSP by evolutionary computation has become a focused study point in current decades in the EC community. However, there are few related studies on the TSP industrial applications, especially in the real industrial products, such as vehicle navigation systems. This paper proposes a TSP solution based on a cost matrix approximation approach and EC acceleration convergence approach in a vehicle navigation system.
There are many research topics in TSP industrial application. In the case of geographic information system, there are two topics, which have be described in this paper. One is the topic on the geographic information data store, design and management for TSP, and the other is the cost matrix approximation algorithm construction, analysis and improvement. Regarding the EC acceleration convergence approach, we used the Lagrange interpolation approach to approximate the EC search space to accelerate EC convergence through an elite obtained approach, and we analyzed the performance of the approach proposed by hypothesis analysis and average convergence comparison.

There are several promising topic, which will be studied in our future work. One is the improvement of the cost matrix approximation approach. We will try to use other geographic information data to improve the cost matrix approximation, which lets the approximated cost matrix as exact as the actual cost. From the actual experimental result, we can also obtain the experimental improvement solution of the geographic information data format. The EC acceleration approach is a main research topic, which includes the interpolation approach selection in different application and implementation, and the acceleration approach evaluation and analysis in the large data set. In our future work we will try to extend the application and implementation area of the proposed approach.

Acknowledgement: This work was supported in part by Grantin-Aid for Scientific Research (23500279). Yan Pei would like to thank Yoshida Scholarship Foundation for its support of his doctoral research.

\section{REFERENCES}

[1] Gupta A., and Bar-Joseph Z., "Extracting Dynamics from Static Cancer Expression Data", IEEE/ACM Trans. on Computational Biology and Bioinformatics, vol.5, no.2, pp.172-182 (Apr.-Jun., 2008). 
[2] Masselos K., Theoharis S., Merakos P., Stouraitis T., and Goutis C.E., "Memory Accesses Reordering for Iinterconnect Power Reduction in Sum-of-products Computations", IEEE Trans. on Signal Processing, vol.50, no.11, pp.2889-2899 (Nov., 2002).

[3] Yang C. L., and Chen Y. K., "Using Genetic Algorithm for Better Route Arrangement a Case of Taiwan Pelican Express Company", IEEE Int. Conf. on Service, Operations, Logistics, Informatics, pp.669-673, Shanghai, China, (21-23, Jun., 2006).

[4] Shima T. ,Rasmussen S., and Gross D., "Assigning Micro UAVs to Task Tours in an Urban Terrain", IEEE Trans. on Control Systems Technology, vol.15, no.4, pp.601-612 (Jul., 2007).

[5] Cheng C.H., Lee W.K., and Wong K.F., "A Genetic Algorithm-based Clustering Approach for Database Partitioning", IEEE Trans. on Syst. Man, Cybern., Part C, vol.32, no.3, pp.215-230 (Aug., 2002).

[6] Lin S., and Kernighan B.W., "An Effective Heuristic Algorithm for the Traveling Salesman Problem", Operations Research, Vol.21, no.2, pp.498-516 (1973).

[7] Yang N., Ma X., and P. Li, "An Improved Angle-Based Crossover Tabu Search the Traveling Salesman Problem", 3rd Int. Conf. on Natural Computation, vol.4, pp.512-516, Haikou, Hainan, China (2007).

[8] Li W., and Alidaee B., "Dynamics of local search heuristics for the traveling salesman problem", IEEE Trans. on Syst. Man, Cybern., Part A, vol.32, no.2, pp.173- 184 (Mar., 2002).

[9] Mahapatra N.R., and Dutt S. , "Scalable Global and Local Hashing Strategies for Duplicate Pruning in Parallel A* Graph Search", IEEE Trans. on Parallel and Distributed Systems, vol.8, no.7, pp.738-756 (Jul., 1997).

[10] Tan K.C., Tang H., and Ge S.S. , "On Parameter Settings of Hopfield Networks Applied to Traveling Salesman Problems", IEEE Trans. on Circuits and Systems I: Regular Papers, vol.52, no.5, pp. 994- 1002 (May., 2005).

[11] Takahashi Y., "A Mathematical Framework for Solving Dynamic Optimization Problems with Adaptive Networks", IEEE Trans. on Syst. Man, Cybern., Part C, vol.28, no.3, pp.404-416 (Aug., 1998).

[12] Takahashi S., Fujimura K., and Tokutaka H., "The SOM-TSP Method for the Three-dimension City Location Problem", Proc. of the 9th Int. Conf. on Neural Information Processing, vol.5, pp.2552-2555, Singapore (18-22, Nov., 2002)

[13] Feng G., and Douligeris C., "Using Hopfield Networks to Solve Traveling Salesman Problems based on Stable State Analysis Technique", Proc. of the IEEE-INNS-ENNS, Int. Joint Conf. on Neural Networks, vol.6, pp.521-526, Como, Italy (24-27, Jul., 2000)

[14] Zhao L., Sun M., Cheng J. H., and Xu Y. Q., "A Novel Chaotic Neural Network With the Ability to Characterize Local Features and Its Application", IEEE Trans. on Neural Networks, vol.20, no.4, pp.735-742 (Apr., 2009).

[15] Saadatmand-Tarzjan M., Khademi M., Akbarzadeh-T. M.-R., and Moghaddam H.A., "A Novel Constructive-Optimizer Neural Network for the Traveling Salesman Problem", IEEE Trans. on Syst. Man, Cybern., Part B, vol.37, no.4, pp.754-770 (Aug., 2007).

[16] Jin H. D., Leung K. S.,Wong M. L., and Xu Z. B., "An Efficient Selforganizing Map Designed by Genetic Algorithms for the Traveling Salesman Problem," IEEE Trans. on Syst. Man, Cybern., Part B, vol.33, no.6, pp.877-888 (Dec., 2003).

[17] Yuyao He, "Chaotic Simulated Annealing with Decaying Chaotic Noise", IEEE Trans. on Neural Networks, vol.13, no.6, pp.1526-1531 (Nov., 2002).

[18] Al-Mulhem M., and Al-Maghrabi T., "Efficient Convex-elastic Net Algorithm to Solve the Euclidean Traveling Salesman Problem," IEEE Trans. on Syst. Man, Cybern., Part B, vol.28, no.4, pp.618620 (Aug., 1998).

[19] Jung S., and Moon B. R., "Toward Minimal Restriction of Genetic Encoding and Crossovers for the Two-dimensional Euclidean TSP", IEEE Trans. on Evolut. Comput., vol.6, no.6, pp.557-565 (Dec., 2002).

[20] Sakurai Y., Takada K., Tsukamoto N., Onoyama T., Knauf R., and Tsuruta S., "Inner Random Restart Genetic Algorithm to Optimize Delivery Schedule", IEEE Trans. on Syst. Man, Cybern., pp.263-270, Istanbul, Turkey (10-13, Oct., 2010).

[21] Hung D. N., Yoshihara I., Yamamori K., and Yasunaga M., "Implementation of an Effective Hybrid GA for Large-Scale Traveling Salesman Problems", IEEE Trans. on Syst. Man, Cybern., Part B, vol.37, no.1, pp.92-99 (Feb., 2007).
[22] Ren S., Wang J., and Zhang X. J., "Research on Chaos ParthenoGenetic Algorithm for TSP", 2010 Int. Conf. on Computer Application and System Modeling, vol.1, pp.290-293, Taiyuan, China (22-24, Oct., 2010)

[23] Mei M., Xue H. F., Zhong M., and Gu Y., "An Improved Differential Evolution Algorithm for TSP Problem", 2010 Int. Conf. on Intelligent Computation Technology and Automation, vol.1, pp.544-547, Changsha, China (11-12, May, 2010).

[24] Vahdati G., Yaghoubi M., Poostchi M., and Naghibi S M.B., "A New Approach to Solve Traveling Salesman Problem Using Genetic Algorithm Based on Heuristic Crossover and Mutation Operator", Int Conf. of Soft Computing and Pattern Recognition 2009, pp.112-116, Malacca, Malaysia (4-7, Dec., 2009).

[25] He Y., Qiu Y. H., Liu G., and Lei K., "A parallel tabu search approach based on genetic crossover operation", 19th Int. Conf. on Advanced Information Networking and Applications, vol.2, pp.467-470, Taipei, Taiwan (28-30, Mar., 2005).

[26] Jiao L. C., and Wang L., "A novel genetic algorithm based on immunity," IEEE Trans. on Syst. Man, Cybern., Part A, vol.30, no.5, pp.552-561 (Sep., 2000).

[27] Cheng C.-H. ,Lee W.-K., and Wong K.-F., "A Genetic Algorithmbased Clustering Approach for Database Partitioning", IEEE Trans. on Syst. Man, Cybern., Part C, vol.32, no.3, pp.215-230 (Aug., 2002).

[28] Shakouri G H., Shojaee K., and Behnam T M., "Investigation on the choice of the initial temperature in the Simulated Annealing: A mushy state SA for TSP", 17th Mediterranean Conf. on Control and Automation 2009, pp.1050-1055, Thessaloniki, Greece (24-26, Jun., 2009).

[29] Yuren Zhou, "Runtime Analysis of an Ant Colony Optimization Algorithm for TSP Instances", IEEE Trans. on Evolut. Comput., vol.13, no.5, pp.1083-1092 (Oct., 2009).

[30] Dorigo M., and Gambardella L.M., "Ant Colony System: a Cooperative Learning Approach to the Traveling Salesman Problem", IEEE Trans. on Evolut. Comput., vol.1, no.1, pp.53-66 (Apr., 1997).

[31] Aras N., Altinel I.K., and Oommen J., "A Kohonen-like Decomposition Method for the Euclidean Traveling Salesman Problem-KNIES DECOMPOSE", IEEE Trans. on Neural Networks, vol.14, no.4, pp. 869-890 (Jul., 2003).

[32] Johnson D.S., and McGeoch L.A., "The Traveling Salesman Problem: a Case Study in Local Optimization", Local Search in Combinatorial Optimization, chapter 8, E.H.L. Aarts and J.K. Lenstra, eds, John Wiley, New York (1997).

[33] Oliver I. M., Smith D. J., and Holland J. R. C., "A Study of Permutation Crossovers on the TSP", in Proc. 2nd Int. Conf. Genetic Algorithm Their Applications, pp.224-230, Cambridge, Ma, USA, (28-31, Jul., 1987).

[34] Muehlenbein H., Gorges-Schleuter M., and Krmer O., "Evolution algorithms in combinatorial optimization", Parallel Comput., vol.7, pp.65-85 (1988).

[35] Nagata Y., and Kobayashi S., "Edge Assembly Crossover: A Highpower Genetic Algorithm for the Traveling SalesmanProblem", in Proc. 7th Int. Conf. Genetic Algorithms, pp.450-457. East Lansing, MI, USA (19-23, Jul., 1997)

[36] Merz P., and Freisleben B., "Memetic Algorithms for the Traveling Salesman Problem", Complex System., vol.13, no.4, pp.297-345 (2001).

[37] Merz P., and Freisleben B., "Genetic Local Search for the TSP: New results", in Proc. IEEE Int. Conf. Evolut. Comput. 1997, pp.159-164, Lake Como, Italy (8-12, Sep. 1997).

[38] Mak K. T., and Morton A. J., "Distances between traveling salesman tours", Discrete Applied Mathematics, vol.58, pp.281-291 (1995).

[39] Pei Y., and Yang G. M., "Application Research on Machine Learning and the Statistic Forecast Algorithm in the Traffic Information Forecast System", vol.2, pp.431-436, 9th Int. Conf. on Hybrid Intelligent Systems, Shenyang, China, (12-14, Aug., 2009).

[40] Pei Y., and Yang G. M., "Algorithms Model of Optimization Path Base on Vector Hyper graph", pp.36-40, Proc. of 14th Youth Conf on Communication, Dalian. China, (24-26, Jul., 2009) (in Chinese).

[41] Pei Y., Cao K., Fu B., Lin Y. J., Chen H. Y., and Xu Y., "A Method and System on Search Waypoint Sequence", China Patent, Application No. CN201110032798.4, Filed on 24 Jan., 2011.

42] Pei Y. and Takagi, H., "Accelerating Evolutionary Computation with Elite Obtained in Projected One-Dimensional Spaces", 5th Int. Conf. on Genetic and Evolutionary Computing, Kimmen Taiwan (ICGEC2011), accepted (Aug. 29-Spt. 1, 2011). 